Georgian Mathematical Journal

1(94), No. 5, 469-478

\title{
PASSAGE OF THE LIMIT THROUGH THE DOUBLE DENJOY INTEGRAL
}

\author{
N. CHELIDZE
}

\begin{abstract}
The conditions are given for passage of the limit through the double Denjoy integral defined by V.G. Chelidze.
\end{abstract}

As is well-known, there exist various definitions of the double Denjoy integral (see $[1,2,5])$. Conditions for passage of limits through these integrals have not yet been studied. The object of this paper is to investigate the conditions for passage of the limit through the double Denjoy integral defined by V.G. Chelidze (see [7]).

Here we shall use the well-known terms (see, for example, [8]). We recall only a few definitions.

Definition 1 (see [8], pp. 127-128). A function $f: R_{0} \rightarrow \mathbb{R}$ with $R_{0}=[a, b] \times[c, d]$ is said to be Denjoy integrable (D-integrable) on $R_{0}$ or, briefly, $f \in D\left(R_{0}\right)$ if there exists a generalized absolutely continuous function $F$ (ACG-function) on $R_{0}$, briefly, $F \in A C G\left(R_{0}\right)$ (see [8], pp. 99$100)$, with an approximate derivative ([8], p. 103) equal to $f$ a.e. The function $F$ is called a $D$-primitive of $f$ and $\Delta\left(f ; R_{0}\right)=F(b, d)-F(a, d)-$ $F(b, c)+F(a, c)$ is called the $D$-integral of $f$ on $R_{0}$ which is written as

$$
\Delta\left(F, R_{0}\right)=(D) \iint_{R_{0}} f(x, y) d x d y .
$$

A function $f: R_{0} \rightarrow \mathbb{R}$ is called $D$-integrable on a measurable ${ }^{1}$ subset $E \subset R_{0}$ (briefly $f \in D(E)$ ), if $f_{E}(x, y) \in D\left(R_{0}\right)$ where

$$
f_{E}(x, y)= \begin{cases}f(x, y) & (x, y) \in E \\ 0 & (x, y) \notin E .\end{cases}
$$

1991 Mathematics Subject Classification. 28A35.

${ }^{1}$ Measurability is meant in the Lebesgue sense and $|A|$ will stand for the Lebesgue measure of the set $A$.

469

1072-947X/94/0900-0469\$07.00/0 @ 1994 Plenum Publishing Corporation 
Definition 2. A compact interval $I$ is said to be of type 2 with respect to $E$ if at least one pair of the opposite vertices of $I$ lies in $E$.

Lemma 1. Let $E_{1}$ be a compact subset of $[a, b]$ whose contiguous intervals are $\left\{r_{i}\right\}_{i}$. If $f \in D(E), f \in D\left(\bar{R}_{i}\right), i=\overline{1, \infty}$, where $E=E_{1} \times[c, d]$, $R_{i}=r_{i} \times[c, d]$, and

$$
\sum_{i=1}^{\infty} O\left(D ; f, \bar{R}_{i}\right)<\infty
$$

where

$$
O\left[D ; f, \bar{R}_{i}\right]=\sup _{\rho \subset \bar{R}_{i}}\left\{\left|(D) \iint_{\rho} f(t, \tau) d t d \tau\right|\right\}
$$

and $\rho$ stands for any measurable subset of $\bar{R}_{i}$. Then $f \in D\left(R_{0}\right)$ and for each subsegment $R \subset R_{0}$ we have

$$
\text { (D) } \iint_{R} f(t, \tau) d t d \tau=(D) \iint_{R \cap E} f(t, \tau) d t d \tau+\sum \sum_{i} \iint_{R \cap \bar{R}_{i}} f(t, \tau) d t d \tau .
$$

Proof. Let us consider the function

$$
F(x, y)=\sum_{i}(D) \iint_{\bar{R}_{i} \cap I(x, y)} f(t, \tau) d t d \tau,
$$

where $I(x, y)=[a, x ; c, y]$.

We shall show that $F$ is continuous on $R_{0}$, i.e., $F$ is continuous at any point $\left(x_{0}, y_{0}\right)$ of $R_{0}$; for $\varepsilon>0$ there must exist a $\delta(\varepsilon)>0$ such that the inequality $\left|F\left(x_{0}+k, y_{0}+l\right)-F\left(x_{0}, y_{0}\right)\right|<\varepsilon$. must take place for all $k$ and $l,|k|<\delta(\varepsilon),|l|<\delta(\varepsilon)$. Then

$$
\begin{aligned}
& \left|F\left(x_{0}+k, y_{0}+l\right)-F\left(x_{0}, y_{0}\right)\right|=\mid \sum_{i}(D) \iint_{\bar{R}_{i} \cap I\left(x_{0}+k, y_{0}+l\right)} f(t, \tau) d t d \tau- \\
& -\sum_{i}(D) \iint_{\bar{R}_{i} \cap I\left(x_{0}, y_{0}\right)} f(t, \tau) d t d \tau\left|\leq \sum_{i}\right|(D) \iint_{\bar{R}_{i} \cap P_{x_{0}, y_{0}}} f(t, \tau) d t d \tau \mid,
\end{aligned}
$$

where $P_{x_{0}, y_{0}}=\left[a, x_{0} ; y_{0}, y_{0}+l\right] \cup\left[x_{0}, x_{0}+k ; c, y_{0}+l\right]$.

To estimate the last sum let us consider two subsystems of intervals from the system $\left\{R_{i}\right\}_{i=1, \infty}: I^{\prime}=\left\{R_{i}^{\prime}\right\}$ and $I^{\prime \prime}=\left\{R_{i}^{\prime \prime}\right\}$ such that system $I^{\prime}$ satisfies the conditions:

$$
\bar{R}_{i}^{\prime} \cap P_{x_{0}, y_{0}} \neq \varnothing \text { and } i>N
$$

and system $I^{\prime \prime}$ the conditions

$$
\bar{R}_{i}^{\prime \prime} \cap P_{x_{0}, y_{0}} \neq \varnothing \text { and } i \leq N \text {. }
$$


For the first system for all $\varepsilon>0$ there is an $N_{0}=N_{0}(\varepsilon)$ such that if $N>N_{0}$, then by the condition (1) we get the estimate

$$
\sum_{i>N}\left|(D) \iint_{\bar{R}_{i}^{\prime} \cap P_{x_{0}, y_{0}}} f(t, \tau) d t d \tau\right|<\sum_{i>N} O\left[D ; f, \bar{R}_{i}^{\prime}\right]<\frac{\varepsilon}{2}
$$

Now consider system $I^{\prime \prime}$. Since $i \leq N$, this system is finite. Let $N^{*}$ be the number of members of this system. Then for $\varepsilon>0$ there is a $\delta(\varepsilon)>0$ such that $\left|P_{x_{0}, y_{0}}\right|<\delta$ and $\left|P_{x_{0}, y_{0}} \cap \bar{R}_{i}^{\prime \prime}\right|<\left|P_{x_{0}, y_{0}}\right|<\delta$. Hence it follows

$$
\text { (D) } \iint_{\bar{R}_{i}^{\prime \prime} \cap P_{x_{0}, y_{0}}} f(t, \tau) d t d \tau<\frac{\varepsilon}{2 N^{*}}
$$

Now

$$
\sum_{i \leq N}\left|(D) \underset{\bar{R}_{i}^{\prime \prime} \cap P_{x_{0}, y_{0}}}{\iint_{i}} f(t, \tau) d t d \tau\right| \leq \frac{\varepsilon}{2 N^{*}} \cdot N^{*}=\frac{\varepsilon}{2} .
$$

Finally, we obtain

$$
\begin{gathered}
\left|F\left(x_{0}+k, y_{0}+l\right)-F\left(x_{0}, y_{0}\right)\right| \leq \sum_{i}\left|(D) \iint_{\bar{R}_{i} \cap P_{x_{0}, y_{0}}} f(t, \tau) d t d \tau\right|= \\
=\sum_{i>N}\left|(D) \iint_{\bar{R}_{i}^{\prime} \cap P_{x_{0}, y_{0}}} f(t, \tau) d t d \tau\right|+\sum_{i \leq N}\left|(D) \iint_{\bar{R}_{i}^{\prime \prime} \cap P_{x_{0}, y_{0}}} f(t, \tau) d t d \tau\right| \leq \varepsilon .
\end{gathered}
$$

Thus $F$ is continuous on $R_{0}$.

Now for the fixed $i$ let us consider any finite system of pairwise disjoint compact intervals of type 2 with respect to $R_{i}$, say, $\left\{S_{k}\right\}_{k=1}^{n}$. If $\sum_{k=1}^{n}\left|S_{k}\right|<$ $\delta$, then, by the conditions of the lemma,

$$
\sum_{k}\left|\Delta\left(F ; S_{k}\right)\right|=\sum_{k}\left|\iint_{S_{k}} f(t, \tau) d t d \tau\right|<\varepsilon
$$

Therefore $F \in A C G\left(\bar{R}_{i}\right)_{i=1, \infty}$. Now we shall show that $F$ is absolutely continuous on $E$ (or, briefly, is $A C$, written as $F \in A C(E)$ ) (see Definition 3 or [8], p. 97). To this end we define the function $G$ as follows:

$$
G(x, y)= \begin{cases}0, & \text { when } \quad(x, y) \in E, \\ \frac{1}{\left|\bar{R}_{i}\right|}(D) \iint_{\bar{R}_{i}} f(t, \tau) d t d \tau, & \text { when } \quad(x, y) \in R_{i}, \quad i=\overline{1, \infty}\end{cases}
$$


We show that $G$ is Lebesgue integrable on $R_{0}$.

$$
\begin{gathered}
\iint_{R_{0}}|G(x, y)| d x d y=\sum_{i} \iint_{\bar{R}_{i}}|G(x, y)| d x d y= \\
=\sum_{i} \iint_{R_{i}}\left(\frac{1}{\left|\bar{R}_{i}\right|}(D)\left|\iint_{\bar{R}_{i}} f(t, \tau) d t d \tau\right|\right) d x d y= \\
=\sum_{i}\left|(D) \iint_{\bar{R}_{i}} f(t, \tau) d t d \tau\right| \iint_{\bar{R}_{i}} \frac{d x d y}{\left|\bar{R}_{i}\right|}=\sum_{i}\left|(D) \iint_{\bar{R}_{i}} f(t, \tau) d t d \tau\right|<C .
\end{gathered}
$$

Put

$$
\Psi(x, y)=\int_{a}^{x} \int_{c}^{y} G(t, \tau) d t d \tau .
$$

Since $G$ is summable, for any $\varepsilon>a$ there is a $\delta>0$ such that $|e|<\delta$ implies

$$
\left|\iint_{e} G(t, \tau) d t d \tau\right|<\varepsilon
$$

for each measurable subset $e \subset R_{0}$.

If we consider any finite system of pairwise disjoint compact intervals of type 2 with respect to $E$, say, $\left\{I_{k}\right\}_{k=1}^{m}$, and if $\sum_{k=1}^{m}\left|I_{k}\right|<\delta$, then

$$
\sum_{k=1}^{m}\left|\iint_{I_{k}} G(t, \tau) d t d \tau\right|<\varepsilon
$$

We shall now show that $\Psi$ is $A C$ on $E$. Assume $I_{k}=\left[\alpha_{k}, \beta_{k} ; \gamma_{k}, \delta_{k}\right]$. Then

$$
\sum_{k=1}^{m}\left|\Delta \Psi\left(I_{k}\right)\right|=\sum_{k=1}^{m}\left|\iint_{I_{k}} G(t, \tau) d t d \tau\right|<\varepsilon .
$$

It is not difficult to check that $F(x, y)=\Psi(x, y)$ when $(x, y) \in E$. Since $F$ is $A C G$ on each $R_{i}$ and $F \in A C(E)$, it follows that $F$ is $A C G$ on $R_{0}$.

Moreover, $D F(x, y)=D \Psi(x, y)=G(x, y)=0$ almost everywhere on $E$ and $D F(x, y)=f(x, y)$ almost everywhere on $R_{0} \backslash E=\bigcup_{i} R_{i}$.

Thus $F$ is a $D$-primitive of $\varphi_{1}$ defined as follows:

$$
\varphi_{1}(x, y)=\left\{\begin{array}{lll}
f(x, y) & \text { when } & (x, y) \in \bigcup_{i} R_{i} \\
0 & \text { when } & (x, y) \in E
\end{array}\right.
$$


On the other hand, we consider

$$
\varphi_{2}(x, y)=\left\{\begin{array}{lll}
0 & \text { when } & (x, y) \in \bigcup_{i} R_{i}, \\
f(x, y) & \text { when } & (x, y) \in E .
\end{array}\right.
$$

Since $f \in D(E)$, we have $\varphi_{2} \in D\left(R_{0}\right)$ and $f \in D\left(R_{0}\right), f(x, y)=\varphi_{1}(x, y)+$ $\varphi_{2}(x, y)$.

Finally, we obtain the equality

$$
\begin{gathered}
(D) \iint_{R} f(t, \tau) d t d \tau=(D) \iint_{R} \varphi_{1}(t, \tau) d t d \tau+(D) \iint_{R} \varphi_{2}(t, \tau) d t d \tau= \\
=(D) \iint_{R \cap E} \varphi_{1}(t, \tau) d t d \tau+(D) \iint_{R \cap\left(\cup_{i} \bar{R}_{i}\right)} \varphi_{1}(t, \tau) d t d \tau+ \\
\quad+(D) \iint_{R \cap E} \varphi_{2}(t, \tau) d t d \tau+(D) \iint_{R \cap\left(\cup_{i} \bar{R}_{i}\right)} \varphi_{2}(t, \tau) d t d \tau= \\
\quad=(D) \iint_{R \cap E} f(t, \tau) d t d \tau+(D) \iint_{R \cap\left(\cup_{i} \bar{R}_{i}\right)} f(t, \tau) d t d \tau= \\
\quad=(D) \iint_{R \cap E} f(t, \tau) d t d \tau+\sum_{i}(D) \iint_{R \cap \bar{R}_{i}} f(t, \tau) d t d \tau,
\end{gathered}
$$

where $R$ is a subsegment of $R_{0}$.

Lemma 2. Let $F_{n}$ be continuous on $R_{0}, n \in N$, and $\lim _{n \rightarrow \infty} F_{n}=F$, where $F$ is also continuous. Then $\lim _{n \rightarrow \infty} O\left[F_{n} ; R_{0}\right] \geq O\left(F ; R_{0}\right)$.

Proof. Since $F$ is continuous, there exist points $\left(x_{1}, y_{1}\right),\left(x_{2}, y_{2}\right)$ on $R_{0}$ such that $F\left(x_{2}, y_{2}\right)-F\left(x_{1}, y_{1}\right)=O\left(F ; R_{0}\right)$. On the other hand, for any $\varepsilon>0$ there is an $N_{0}=N_{0}(\varepsilon)>0$ such that $F_{n}\left(x_{i}, y_{i}\right)-\varepsilon \leq F\left(x_{i}, y_{i}\right) \leq$ $F_{n}\left(x_{i}, y_{i}\right)+\varepsilon, i=1,2$, for all $n>N_{0}$.

It follows that $F\left(x_{2}, y_{2}\right)-F\left(x_{1}, y_{1}\right) \leq F_{n}\left(x_{2}, y_{2}\right)-F_{n}\left(x_{1}, y_{1}\right)+2 \varepsilon$ and so $O\left(F ; R_{0}\right) \leq O\left(F_{n} ; R_{0}\right)+2 \varepsilon$ from which the result is immediate.

Definition 3. Let $F_{n}: R_{0} \rightarrow R$ be continuous, $n \in N$. Then $\left\{F_{n} ; n \in\right.$ $N\}$ is said to be a sequence of uniformly $A_{x}$-functions on the set $P \subset[a, b]$ if $P=\bigcup_{k} P_{k}$ and $F_{n}$ is uniformly $A C$ on each $H_{k}=P_{k} \times[c, d]$ (see [9]), i.e., for all $\varepsilon>0$ there is a $\delta=\delta(\varepsilon)>0$ such that for any finite system of pairwise disjoint compact intervals of type 2 with respect to $H_{k}$, say, $\left\{R_{i}\right\}$, $i=\overline{1, m}$, the inequality $\sum_{i=1}^{m}\left|R_{i}\right|<\delta$ implies $\sum_{i=1}^{m}\left|\Delta\left(F_{n} ; R_{i}\right)\right|<\varepsilon$ for all $n \in N$.

Lemma 3. If $f_{n} \in D\left(R_{0}\right), n \in N, E \subset[a, b]$ is a closed set and the sequence

$$
F_{n}(x, y)=\int_{a}^{x} \int_{c}^{y} f_{n}(t, \tau) d t d \tau, \quad n \in N,
$$


is uniformly $A C$ on $E^{\prime}=E \times[\gamma, \delta]$, then for all $\varepsilon>0$ there is a $\delta=\delta(\varepsilon)>0$ such that for all measurable sets, $e, e \subset E$ and $|e|<\delta$, we have

$$
\left|\iint_{e^{\prime}} f_{n}(t, \tau) d t d \tau\right|<\varepsilon, \quad n=\overline{1, \infty}, \text { where } e^{\prime}=e \times[\gamma, \delta] .
$$

Proof. By the conditions of the lemma, if $\varepsilon>0$, then there is a $\delta>0$ such that for any finite system $\left\{R_{k}\right\}, k=\overline{1, m}$, of disjoint compact intervals of type 2 with respect to $E^{\prime}$ the inequality $\sum_{k=1}^{m}\left|R_{k}\right|<\delta$ implies

$$
\sum_{k=1}^{m}\left|F_{n}\left(R_{k}\right)\right|=\sum_{k=1}^{m}\left|\iint_{R_{k}} f_{n}(t, \tau) d t d \tau\right|<\varepsilon
$$

for all $n \in N$.

Let now $e$ be a measurable subset of $E$ with $|e|<\delta$. Then there are open sets $G_{m}, m \in N$, such that $G_{m} \supset G_{m+1} \supset e, m \in N$, and $\lim _{m \rightarrow \infty}\left|G_{m}\right|=$ $|e|$. It can further be assumed that $G_{m}=\cup_{k=1}^{\infty} r_{k}^{(m)}, r_{k}^{(m)} \cap r_{e}^{(m)}=\varnothing$, $k \neq l$, for each $m$, where $r_{k}^{(m)}$ are open intervals. We set $e_{m}=E \cap G_{m}=$ $\cup_{k}\left(E \cap r_{k}^{(m)}\right)$. Denote by $\rho_{k, j}^{(m)}, j \in N$, contiguous intervals of $E \cap r_{k}^{(m)}$ on $r_{k}^{(m)}$. Then $G_{m} \backslash e_{m}=\cup_{k} \cup_{j} \rho_{k, j}^{(m)}$.

The endpoints of each $\rho_{k, j}^{(m)}$ lie on $E$. Moreover, for $\delta=\delta(\varepsilon)>0$ there is an $N_{0}(\delta)=N_{0}>0$ such that if $m>N_{0}$, then $\left|G_{m}\right|<\delta$.

If $m>N_{0}$, from (3) we obtain

$$
\begin{gathered}
\sum_{k}\left|\iint_{r_{k}^{(m)} \times[\gamma, \delta]} f_{n}(t, \tau) d t d \tau\right|<\varepsilon, \\
\sum_{k} \sum_{j}\left|\iint_{\rho_{k, j}^{(m)} \times[\gamma, \delta]} f_{n}(t, \tau) d t d \tau\right|<\varepsilon
\end{gathered}
$$

for all $n \in N$.

Now, by Lemma 1 , for $m>N_{0}$ we have

$$
\begin{gathered}
\iint_{r_{k}^{(m)} \times[\gamma, \delta]} f_{n}(t, \tau) d t d \tau= \\
=\iint_{\left(r_{k}^{(m)} \cap E\right) \times[\gamma, \delta]} f_{n}(t, \tau) d t d \tau+\sum_{j} \iint_{\rho_{k, j}^{(m)} \times[\gamma, \delta]} f_{n}(t, \tau) d t d \tau, \quad n=\overline{1, \infty} .
\end{gathered}
$$

Performing the summation of the last expression over $k$, from (4) we obtain

$$
\left|\iint_{e_{m} \times[\gamma, \delta]} f_{n}(t, \tau) d t d \tau\right|<2 \varepsilon, \quad n=\overline{1, \infty}
$$


and so

$$
\left|\iint_{e^{\prime}} f_{n}(t, \tau) d t d \tau\right|<2 \varepsilon, \quad \overline{1, \infty} .
$$

Note that the following lemma of P. Romanovskii is very essential for our investigation.

Lemma 4. Let $\mathfrak{F}$ be a nonempty system of open subintervals of the open interval $] a, b[$, having the following four properties:

1) If $] \alpha, \beta[\in \mathfrak{F}$ and $] \beta, \gamma[\in \mathfrak{F}$, then $] \alpha, \gamma[\in \mathfrak{F}$;

2) If $] \alpha, \beta[\in \mathfrak{F}$, then every subinterval of $] \alpha, \beta[$ also belongs to $\mathfrak{F}$;

3) If every proper open subinterval of $] \alpha, \beta[$ belongs to $\mathfrak{F}$, then $] \alpha, \beta[\in \mathfrak{F}$;

4) If all contiguous intervals on $[a, b]$ of a nonempty perfect set $E$ from ]$a, b[$ belong to $\mathfrak{F}$, then $\mathfrak{F}$ contains an interval $] \alpha, \beta[$ such that $] \alpha, \beta[\cap E \neq \varnothing$.

Then $] a, b[\in \mathfrak{F}$.

Theorem. If $f_{n} \in D\left(R_{0}\right), \lim _{n \rightarrow \infty} f_{n}=f$ almost everywhere on $R_{0}$ and the sequence

$$
F_{n}(x, y)=\int_{a}^{x} \int_{c}^{y} f_{n}(t, \tau) d t d \tau, \quad n=\overline{1, \infty}
$$

is uniformly continuous on $R_{0}$ and uniformly $A_{x}$ on $[a, b]$, then $f \in D\left(R_{0}\right)$ and

$$
\lim _{n \rightarrow \infty} \iint_{R_{0}} f_{n}(t, \tau) d t d \tau=\iint_{R_{0}} f(t, \tau) d t d \tau .
$$

Proof. The subinterval $] \alpha, \beta[\subset] a, b[$ will be said to lie in the family of subintervals $\mathfrak{F}$ if for $] \alpha, \beta[$ and all all compact subintervals we have

$$
\lim _{n \rightarrow \infty} \int_{\alpha}^{\beta} \int_{\gamma}^{\delta} f_{n}(t, \tau) d t d \tau=\int_{\alpha}^{\beta} \int_{\gamma}^{\delta} f(t, \tau) d t d \tau,
$$

where $[\gamma, \delta] \subset[c, d]$.

The theorem will be proved if we show that $\mathfrak{F}$ satisfies the conditions of Romanovskii's lemma. The condition of the theorem implies that there exists a portion $P$ such that $\left\{F_{n}\right\}_{n \geq 1}$ is uniformly $A C$ on $P \times[c, d]$. Then due to Vitali's theorem on passage of limits through integrals and by virtue of some properties of the Denjoy integral there exists an interval $\left[\alpha^{*}, \beta^{*}, \gamma^{*}, \delta^{*}\right]$ for which the equation $\left(^{*}\right)$ is fulfilled. Thus $\mathfrak{F} \neq \varnothing$. 
Now assume that $] \alpha, \beta[\in \mathfrak{F}$ and $] \beta, \eta[\in \mathfrak{F}$. Then $] \alpha, \eta[\in \mathfrak{F}$. Indeed,

$$
\begin{gathered}
\lim _{n \rightarrow \infty} \int_{\alpha}^{\eta} \int_{\gamma}^{\delta} f_{n}(t, \tau) d t d \tau=\lim _{n \rightarrow \infty}\left(\int_{\alpha}^{\beta} \int_{\gamma}^{\delta} f_{n}(t, \tau) d t d \tau+\int_{\beta}^{\eta} \int_{\gamma}^{\delta} f_{n}(t, \tau) d t d \tau\right)= \\
=\int_{\alpha}^{\beta} \int_{\gamma}^{\delta} f(t, \tau) d t d \tau+\int_{\beta}^{\eta} \int_{\gamma}^{\delta} f(t, \tau) d t d \tau=\int_{\alpha}^{\eta} \int_{\gamma}^{\delta} f(t, \tau) d t d \tau,
\end{gathered}
$$

i.e., $] \alpha, \eta[\in \mathfrak{F}$.

Condition 2) follows from the definition of the family $\mathfrak{F}$.

Now let us consider condition 3 ). It is assumed that every open proper subinterval of $] \alpha, \beta[$ is contained in $\mathfrak{F}$. Then we must show that $] \alpha, \beta[\in \mathfrak{F}$.

Consider $] \lambda_{m}, \mu_{m}[\in \mathfrak{F},] \lambda_{m}, \mu_{m}[\subset] \alpha, \beta\left[, \lambda_{m} \downarrow \alpha, \mu_{m} \uparrow \beta, m \rightarrow \infty\right.$.

Since $\left\{F_{n}\right\}_{n \geq 1}$ is uniformly continuous on $R_{0}$, for all $\varepsilon>0$ there is an $N_{0}=N_{0}(\varepsilon)$ such that if $m>N_{0}$, then

$$
\int_{\lambda_{m}}^{\mu_{m}} \int_{\gamma}^{\delta} f_{n}(t, \tau) d t d \tau-\varepsilon \leq \int_{\alpha}^{\beta} \int_{\gamma}^{\delta} f_{n}(t, \tau) d t d \tau \leq \int_{\lambda_{m}}^{\mu_{m}} \int_{\gamma}^{\delta} f_{n}(t, \tau) d t d \tau+\varepsilon,
$$

where $[\gamma, \delta] \subset[c, d]$ is arbitrary.

Since $] \lambda_{m}, \mu_{m}[\in \mathfrak{F}$, we have

$$
\begin{aligned}
& \int_{\lambda_{m}}^{\mu_{m}} \int_{\gamma}^{\delta} f(t, \tau) d t d \tau-\varepsilon \leq \underline{\lim _{n \rightarrow \infty}} \int_{\alpha}^{\beta} \int_{\gamma}^{\delta} f_{n}(t, \tau) d t d \tau \leq \\
& \leq \varlimsup_{n \rightarrow \infty} \int_{\alpha}^{\beta} \int_{\gamma}^{\delta} f_{n}(t, \tau) d t d \tau \leq \int_{\lambda_{m}}^{\mu_{m}} \int_{\gamma}^{\delta} f(t, \tau) d t d \tau+\varepsilon .
\end{aligned}
$$

Hence, $\varepsilon>0$ being arbitrary, (5) implies that

$$
\lim _{n \rightarrow \infty} \int_{\lambda_{m}}^{\mu_{m}} \int_{\gamma}^{\delta} f(t, \tau) d t d \tau
$$

exists and

$$
\lim _{n \rightarrow \infty} \int_{\alpha}^{\beta} \int_{\gamma}^{\delta} f_{n}(t, \tau) d t d \tau=\int_{\alpha}^{\beta} \int_{\gamma}^{\delta} f(t, \tau) d t d \tau
$$

Thus $] \alpha, \beta[\in \mathfrak{F}$ and condition 3$)$ is satisfied. 
Finally, we shall check condition 4). It is assumed that $E \subset[a, b]$ is a closed set. Since the sequence $\left\{F_{n}\right\}_{n \geq 1}$ is uniformly $A_{x}$ on $[a, b]$, there exists a portion $P=E \cap[\alpha, \beta]$ such that $\left\{F_{n}\right\}_{n \geq 1}$ is uniformly $A C$ on $P \times[c, d]$.

Let $\left\{I_{k}=\right] \alpha_{k}, \beta_{k}[, k=\overline{1, \infty}\}$ be the contiguous intervals of $P$ on $] \alpha, \beta[$ which are the members of $\mathfrak{F}$. By Lemma 1 , where $I_{k} \times[\gamma, \delta]$ are the contiguous intervals of $P \times[\gamma, \delta], k=\overline{1, \infty}$, we have

$$
\int_{\alpha}^{\beta} \int_{\gamma}^{\delta} f_{n}(t, \tau) d t d \tau=\iint_{P \times[\gamma, \delta]} f_{n}(t, \tau) d t d \tau+\sum_{k} \iint_{I_{k} \times[\gamma, \delta]} f_{n}(t, \tau) d t d \tau
$$

By Lemma 3 and Vitali's theorem on passage of limits through integrals

$$
\lim _{n \rightarrow \infty} \iint_{P^{\prime}} f_{n}(t, \tau) d t d \tau=\iint_{P^{\prime}} f(t, \tau) d t d \tau,
$$

where $P^{\prime}=P \times[\gamma, \delta]$.

Since $\left\{F_{n}\right\}_{n \geq 1}$ is uniformly $A C$ on $P^{\prime}$ for all $\varepsilon>0$, there is an $N_{0}=N_{0}(\varepsilon)$ such that if $k>N_{0}$, then

$$
\sum_{k>N_{0}} O\left(F_{n} ; I_{k} \times[\gamma, \delta]\right)<\varepsilon .
$$

Since $I_{k} \in \mathfrak{F}$, (9) implies

$$
\sum_{k>N_{0}} O\left(F ; I_{k} \times[\gamma, \delta]\right)<\varepsilon, \sum_{k=1}^{\infty} O\left(F ; I_{k} \times[\gamma, \delta]\right)<+\infty .
$$

Thus $f \in D([\alpha, \beta ; \gamma, \delta])$ and from (7), (8), (10) we obtain

$$
\lim _{n \rightarrow \infty} \int_{\alpha}^{\beta} \int_{\gamma}^{\delta} f_{n}(t, \tau) d t d \tau=\int_{\alpha}^{\beta} \int_{\gamma}^{\delta} f(t, \tau) d t d \tau
$$

i.e., $] \alpha, \beta[\in \mathfrak{F}$ and $] \alpha, \beta[\cap E \neq \varnothing$. Therefore $\mathfrak{F}$ satisfies all conditions of Romanovskii's lemma and $] a, b[\in \mathfrak{F}$.

\section{REFERENCES}

1. M. Krzyzánski, Sur l'extension de l'opération integrale de Denjoy aux fonctions de deux variables. Bull. du Séminaire Math. de l'université de Wilno 2(1939), 41-51.

2. S. Kempisty, Sur les fonctions absolument continues d'intervalle. Fund. Math. 27(1937), 10-37. 
3. S. Kempisty, Sur les fonctions absolument semi-continues. Fund. Math. 30(1938), 104-127.

4. H. Looman, Sur la totalisation des děrivées des fonctions de plusieurs variables independant. Fund. Math. 4(1923), 246-285.

5. P.L. Romanovskii, Intégrale de Denjoy dans les espaces abstraits. Mat. Sb. 9(1941), 67-120.

6. G.P. Tolstov, On curvilinear and repeated integral. (Russian) Trudy Matem. Inst. Steklova, 35(1950), 1-100.

7. V.G. Chelidze, Double integrals of Denjoy. (Russian) Trudy Tbiliss. Mat. Inst. Razmadze, 15(1947), 155-242.

8. V.G. Chelidze and A.G. Djvarsheishvili, The theory of the Denjoy integral and some applications. (Translated from the Russian by P.S. Bullen) World Scientific, Singapore, New Jersey, London, Hong Kong, 1989; Russian original: Tbilisi University Press, Tbilisi, 1978.

9. A. G. Djvarsheishvili, On the integration of the product of two functions. (Russian) Trudy Tbiliss. Mat. Inst. Razmadze, 24(1957), 3551.

(Received 28.08.1992, revized version 25.06.1993)

Author's address:

Faculty of Mechanics and Mathematics

I. Javakhishvili Tbilisi State University

2, University st., Tbilisi 380043

Republic of Georgia 